\title{
MINIMAL SURFACES AND $H$-SURFACES IN NONPOSITIVELY CURVED SPACE FORMS
}

\author{
BENNETT PALMER
}

(Communicated by Peter Li)

\begin{abstract}
We show that if the Gauss curvature of a surface of constant mean curvature in a nonpositively curved space form is sufficiently pinched, the surface is stable. In this case, we also give an upper bound for the inradius. We then show that the inradius of a stable minimal surface in Euclidean space, which is contained in a solid cylinder, is bounded above by a constant depending only on the radius of the cylinder.
\end{abstract}

Let $M^{3}(c)$ denote a 3-dimensional oriented space form of constant sectional curvature $c \leq 0$. Let $X: M \rightarrow M^{3}(c)$ be a smooth immersion of a smoothly bounded surface $M$ with curvature $K$ and mean curvature $h$. Set

$$
\bar{K}=\max _{M} K, \quad \underline{K}=\min _{M} K .
$$

We show

Theorem I. For $h, c \in \mathbb{R}$ with $-A^{2}:=h^{2}+c \leq 0$, there exist universal constants $\omega(c, h) \geq e^{2}$ with the following property: $h$ and

If $M \subset M^{3}(c)$ is a smooth orientable surface with constant mean curvature

$$
\left(-A^{2}-\underline{K}\right) /\left(-A^{2}-\bar{K}\right) \leq \omega(c, h)
$$

then $M$ is stable. In addition, $7.4 \ldots=e^{2} \leq \omega(0,0) \leq 10.75 \ldots$ holds.

Theorem II. If $-\infty<\underline{K}, \bar{K}<-A^{2}$, and $M$ contains a geodesic ball $B_{r}\left(x_{0}\right)$ of radius $r$ then

$$
r^{2} \leq \frac{\pi^{2}}{4\left(-A^{2}-\bar{K}\right)} \log _{e}\left[\left(-A^{2}-\underline{K}\right) /\left(-A^{2}-\bar{K}\right)\right] .
$$

In the second part of this paper we consider surfaces in $\mathbb{E}^{N}$ which are extrinsically bounded in some way. Let $C_{R}=\left\{X=\left(X_{1}, X_{2}, X_{3}\right) \in \mathbb{E}^{3} \mid x_{1}^{2}+x_{2}^{2}<\right.$ $R^{2}$. We show

Theorem III. There exists a constant $c_{1}>0$ with the following property: If $M \subset$ $\mathbb{E}^{3}$ is an orientable stable minimal surface with $B_{r}(p) \subset M$ and $M \subset C_{R_{1}} \backslash C_{R_{2}}$ for some $R_{1}>R_{2} \geq 0$ then $r^{2} \leq c_{1}\left(R_{1}^{2}-R_{2}^{2}\right)$.

Received by the editors September 11, 1991.

1991 Mathematics Subject Classification. Primary 53A10. 
Before beginning the proofs, we review some basic facts about surfaces in $M^{3}(c)$.

Let $M \rightarrow M^{3}(c)$ be an orientable surface. Let $d s^{2}$ denote the induced metric. $\left(M, d s^{2}\right)$ may be considered a Riemann surface in a natural way by introducing isothermal coordinates $(x, y)$ and using $z=x+i y$ as a complex coordinate. Doing so, $d s^{2}$ may be expressed as $d s^{2}=e^{\rho}|d z|^{2}$ and the curvature is

$$
K=-2 e^{-\rho} \rho_{z \bar{z}} .
$$

The second fundamental form of $M$ has an expression

$$
\Pi=\operatorname{Re}\left\{\phi d z^{2}+h e^{\rho} d z d \bar{z}\right\}
$$

where $\phi d z^{2}$ is an invariant quadratic differential and $h$ is the mean curvature. The fundamental equations of the immersion are those of Gauss

$$
|\phi|^{2}=e^{2 \rho}\left(h^{2}+c-K\right)
$$

and Codazzi

$$
\phi_{\bar{z}}=e^{\rho} h_{z} .
$$

When $h=$ const, (3) implies that $\phi$ is holomorphic in $z$. It then follows that either $\phi \equiv 0$ and $M$ is totally umbilic or the zeros of $\phi$ are isolated.

Lemma 1. Let $M \subset M^{3}(c), c \leq 0$, have constant mean curvature $h$ such that $h^{2}+c \leq 0$. Assume $M$ has no umbilics. Then the conformal metric

$$
d \tilde{s}^{2} \equiv\left(h^{2}+c-K\right) d s^{2}
$$

has curvature $\widetilde{K}$ satisfying

$$
\widetilde{K} \geq 1 \text {. }
$$

Proof. Using (1)-(3) one has

$$
\begin{aligned}
0 & =\Delta \log |\phi|=\Delta \log \left(-A^{2}-K\right)^{1 / 2}+\Delta \rho \\
& =\Delta \log \left(-A^{2}-K\right)^{1 / 2}-2 K
\end{aligned}
$$

where $\Delta=4 e^{-\rho} \partial z \partial \bar{z}$. Therefore, using (1) to compute $\widetilde{K}$ one has

$$
\widetilde{K}=-\left(-A^{2}-K\right)^{-1}\left\{\Delta \log \left(-A^{2}-K\right)^{1 / 2}-K\right\}=\left(-A^{2}-K\right)^{-1}(-K) .
$$

Since $K<0$ on $M,(5)$ follows.

Proposition 1. Again assume $h=\mathrm{const}, h^{2}+c \equiv-A^{2} \leq 0$, and

$$
0 \leq-A^{2}-\bar{K} \leq-A^{2}-K \leq-A^{2}-\underline{K}<\infty .
$$

Then the first (nontrivial) eigenvalue $\lambda_{1}$ of the problem

$$
\begin{cases}\Delta \psi+2 \lambda\left(-A^{2}-K\right) \psi=0 & \text { on } M, \\ \psi=0 & \text { on } \partial M\end{cases}
$$

satisfies

$$
\left[\frac{1}{2} \log \left(\left(-A^{2}-\underline{K}\right) /\left(-A^{2}-\bar{K}\right)\right)\right]^{-1} \leq \lambda_{1} .
$$


Proof. Let $\psi \geq 0$ be the eigenfunction corresponding to $\lambda_{1}$. Let $g(x, y)$ denote the positive Green's function of $M$. Then

$$
\begin{aligned}
\psi(x) & =\lambda_{1} \int_{\Omega}\left(-2 A^{2}-2 K(y)\right) \psi(y) g(x, y) * 1(y) \\
& \leq \lambda_{1} \int_{\Omega}-2 K(y) \psi(y) g(x, y) * 1(y) .
\end{aligned}
$$

Therefore,

$$
|\psi(y)| \leq \lambda_{1}\|\psi\|_{\infty} \int-2 K(y) g(x, y) * 1(y) \equiv \lambda_{1}\|\psi\|_{\infty} \nu(x),
$$

where $\nu$ solves

$$
\begin{array}{rlrl}
\Delta \nu & =2 K & & \text { in } M, \\
\nu \equiv 0 & & \text { on } \partial M .
\end{array}
$$

Choosing $x$ where $\psi$ achieves its maximum we arrive at

$$
1 \leq \lambda_{1}\|\nu\|_{\infty}
$$

By (6) and (9)

$$
\Delta\left(\nu-\log \left(-A^{2}-K\right)^{1 / 2}\right)=0 \quad \text { in } M
$$

and on $\partial M$

$$
\nu-\log \left(-A^{2}-K\right)^{1 / 2} \leq-\log \left(-A^{2}-\bar{K}\right)^{1 / 2} .
$$

It follows from the maximum principle and (8) that on $M$

$$
\begin{aligned}
\nu & \leq \log \left(-A^{2}-K\right)^{1 / 2}-\log \left(-A^{2}-\bar{K}\right)^{1 / 2} \\
& \leq \log \left(-A^{2}-\underline{K}\right)^{1 / 2}-\log \left(-A^{2}-\bar{K}\right)^{1 / 2} .
\end{aligned}
$$

Using this and (10), (8) follows.

Proof of Theorem I. The surface $M$ is stationary for the functional

$$
J=\text { area }+2 H \text { (enclosed 3-volume). }
$$

The second variation of $J$ for variations of the form $\psi \cdot N$ where $N$ is the unit normal to $M$ and $\psi \in C_{0}^{\infty}(M)$ is given by $\delta^{2} J=\int-\psi L \psi$. Here $L$ is the selfadjoint elliptic operator $L \psi=\Delta \psi+2\left(-2 A^{2}-K\right) \psi$. Assuming the hypothesis of the theorem, we have by Proposition $1, \lambda_{1} \geq 1$. Using integration by parts we obtain

$$
\begin{aligned}
\delta^{2} J & =\int-\psi L \psi=\int\left(|\nabla \psi|^{2}-2\left(-2 A^{2}-K\right) \psi^{2}\right) \\
& \geq \int\left(|\nabla \psi|^{2}-2\left(-A^{2}-K\right) \psi^{2}\right) \geq 0
\end{aligned}
$$

and $M$ is stable.

The upper bound for $\omega(0,0)$ follows from Example I.

Remark. We have shown that under the hypothesis of Theorem I, the second variation of $J$ is nonnegative for all compactly supported variations. When $h \neq 0$ this is stronger than the condition that $\delta^{2} J$ be nonnegative for all volume-preserving variations (cf. [B-DC]). 
Example I. Let $C \subset \mathbb{R}^{3} \approx \mathbb{C} \times \mathbb{R}$ be the catenoid parameterized by $X(u, v)=$ $\left(e^{i v} \cosh (u), u\right),(u, v) \in \mathbb{R} \times[0,2 \pi)$. One easily computes that the curvature is given by $K=-(\cosh u)^{-4}$ and that the support function is given by $s=$ $-1+u \tanh (u)$.

Denote by $\Omega_{t}$ the symmetric "waist" domain of the catenoid given by $|u|<$ $t$. Then $\Omega_{t}$ will be stable as long as $s$ is negative, that is, for $t<u_{1} \approx 1.2 \ldots$. For $\Omega_{t}$

$$
e^{2} \geq \underline{K} / \bar{K}=(\cosh t)^{4}
$$

holds for $t<t_{1} \approx 1.0850 \ldots$. It follows that Theorem I has correctly predicted stability in this case. Furthermore, for $\Omega u_{1}, \underline{K} / \bar{K} \approx 10.75 \ldots$ furnishes the upper bound in the corollary. Finally the total curvature of $\Omega_{t}$ is

$$
2 \pi \int_{-t_{1}}^{t_{1}} \cosh ^{-2} u d u \approx 2 \pi(1.590 \ldots)>2 \pi .
$$

Consequently the criteria of Theorem I is independent of the Barbosa-DoCarmo result [B-DC1].

To prove Theorem II we state without proof a special case of an eigenvalue estimate due to Gage [G].

Theorem (Gage). Let $\widetilde{B}_{\tilde{r}}$ be a geodesic ball of radius $\tilde{r}$ contained in a surface of curvature $\widetilde{K} \geq-\beta^{2}=$ const. Then the first Dirichlet eigenvalue of the Laplacian $\widetilde{\Delta}$ on $\widetilde{B}_{r}$ satisfies

$$
\tilde{\lambda}_{1} \leq \pi^{2} / \tilde{r}^{2}+\beta^{2} / 4
$$

Proof of Theorem II. For a region $\Omega \subset M$ let $\tilde{\lambda}_{1}(\Omega)$ denote the first Dirichlet eigenvalue of $\tilde{\Delta}$ for $\Omega$. Here $\tilde{\Delta}$ is the Laplacian for the metric $d \tilde{s}$ of Lemma 1. Let $\lambda_{1}(\Omega)$ be the first eigenvalue of the problem

$$
\begin{aligned}
\Delta \psi+2 \lambda\left(-A^{2}-K\right) \psi & =0 & & \text { in } \Omega, \\
\psi & =0 & & \text { on } \partial \Omega .
\end{aligned}
$$

Since $\widetilde{\Delta}=\left(-A^{2}-K\right)^{-1} \Delta$, it is clear that $\tilde{\lambda}_{1}(\Omega)=2 \lambda_{1}(\Omega)$. Let $\gamma$ be a minimizing geodesic of length $\tilde{r}$ for the metric $d \tilde{s}$. Then

$$
\tilde{r}=\int_{\gamma}\left(-A^{2}-K\right)^{1 / 2} d s \geq\left(-A^{2}-\bar{K}\right)^{1 / 2} \int_{\gamma} d s .
$$

It follows that $\widetilde{B}_{r\left(-A^{2}-\bar{K}\right)^{1 / 2}} \subset B_{r}$. By a well-known monotonicity property of eigenvalues

$$
\tilde{\lambda}_{1}\left(\tilde{B}_{r\left(-A^{2}-\bar{K}\right)^{1 / 2}}\right) \geq \tilde{\lambda}_{1}\left(B_{r}\right)=2 \lambda_{1}\left(B_{r}\right) .
$$

So by Lemma 1 and Gage's Theorem with $\beta=0$, we have

$$
\frac{\pi^{2}}{r^{2}\left(-A^{2}-\bar{K}\right)} \geq 2 \lambda_{1}\left(B_{r}\right) \text {. }
$$

Combining this with the lower bound of Proposition 1 yields the result.

We now consider surfaces in $\mathbb{E}^{N}$ which are extrinsically bounded. 
Lemma 2. Let $M \subset \mathbb{E}^{3}$ be a minimal surface. Let $\Omega \subset M$ be a smoothly bounded subdomain, and let $\mu_{1}$ be the first Dirichlet eigenvalue of the Laplacian in $\Omega$. Assume

$$
M \subset C_{R_{1}} \backslash C_{R_{2}}, \quad R_{1}>R_{2} \geq 0 .
$$

Then

$$
\mu_{1} \geq \frac{2}{R_{1}^{2}-R_{2}^{2}}
$$

Proof. Define $\tau=\frac{1}{2}\left(x_{1}^{2}+x_{2}^{2}\right)$. Let $N=\left(N_{1}, N_{2}, N_{3}\right)$ be a unit normal defined on a neighborhood in $M$. Then

$$
\begin{aligned}
\Delta \tau & =\frac{1}{2} \sum_{i=1,2}\left(2 x_{i} \Delta x_{i}+2\left\|\nabla x_{i}\right\|^{2}\right)=\left\|\nabla x_{1}\right\|^{2}+\left\|\nabla x_{2}\right\|^{2} \\
& =1-N_{1}^{2}+1-N_{2}^{2}=1+N_{3}^{2} \geq 1 .
\end{aligned}
$$

By (14) we have

$$
\frac{R_{2}^{2}}{2}<\tau<\frac{R_{1}^{2}}{2} .
$$

Let $\psi \geq 0$ be a solution of $\Delta \psi+\mu \psi=0$ with $\psi \equiv 0$ on $\partial \Omega$. Then

$$
\psi(x)=\mu_{1} \int_{\Omega} \psi(y) g(x, y) * 1(y)
$$

and consequently

$$
|\psi(x)| \leq \mu_{1}\|\psi\|_{\infty} \int_{\Omega} g(x, y) * 1(y) .
$$

Taking $x$ values where $\psi$ achieves its maximum yields

$$
1 \leq \mu_{1} \max _{x \in \Omega} \int_{\Omega} g(x, y) * 1(y) \equiv \mu_{1} \max _{x \in \Omega} S(x)
$$

where $S$ solves

$$
\begin{aligned}
\Delta S & =-1 & & \text { in } \Omega, \\
S & \equiv 0 & & \text { on } \partial \Omega .
\end{aligned}
$$

Therefore,

$$
\Delta(S+\tau) \geq 0 \quad \text { in } \Omega \quad \text { and } \quad S+\tau=\tau \leq R_{1}^{2} / 2 \quad \text { on } \partial \Omega .
$$

By the maximum principle

$$
S \leq \frac{R_{1}^{2}}{2}-\tau \leq \frac{R_{1}^{2}}{2}-\frac{R_{2}^{2}}{2} \text { in } \Omega .
$$

Combining this with (17) proves (15).

Proof of Theorem III. Since $M$ is stable, it follows by a result of Schoen [Sc, Corollary 4] that there is an estimate $K(x) \geq-2 \alpha / r^{2}$, for all $x \in B_{r / 2}$, where $\alpha$ is a universal constant. Using this lower bound for $K$ in Gage's upper bound for $\mu_{1}$ gives

$$
\mu_{1} \leq \frac{\alpha}{4 r^{2}}+\frac{\pi^{2}}{r^{2}}=: \frac{4 C_{1}}{r^{2}} .
$$

Combining this with the lower bound for $\mu_{1}$ in Lemma 2 gives the result. 
Corollary. Let $M \subset \mathbb{E}^{3}$ be a complete minimal surface. If there exists $p \in M$ such that

$$
\limsup _{r \rightarrow \infty} \frac{1}{r} \int_{B_{r}(P)}(-K)=0
$$

then $M$ is not contained in a cylinder.

Proof. Assume to the contrary that $M \subset C_{R}$ for some $R, 0<R<\infty$. Let $r_{0}$ be a constant with $r_{0}^{2}>c_{1} R^{2}$ with $c_{1}$ as in Theorem III. Then any disc $B_{r_{0}} \subset M$ is unstable. By a result of Barbosa and DoCarmo [B-DC1] $\int_{B_{r_{0}}}(-K)>2 \pi$. Taking the sequence $r_{n}=n r_{0}$, one finds that since $B_{r_{n}}(p)$ contains at least $n$ disjoint geodesic balls of radius $r_{0}$,

$$
\frac{1}{r_{n}} \int_{B_{r_{n}}(P)}(-K)>\frac{2 \pi n}{n r_{0}}=\frac{2 \pi}{r_{0}} \gg 0
$$

giving a contradiction.

\section{ACKNOWLEDGMENT}

We would like to thank Professor Robert Osserman for his helpful comments.

\section{REFERENCES}

[B-DC] J. L. Barbosa and M. DoCarmo, Stability of hypersurfaces with constant mean curvatures, Math. Z. 185 (1984), 339-353.

[B-DC1] _ On the size of a stable minimal surface in $\mathbb{R}^{3}$, Amer. J. Math. 98 (1976), 515-528.

[F-C-S] D. Fischer-Colbrie and R. Schoen, The structure of complete stable, minimal surfaces in 3-manifolds of non-negative scalar curvature, Comm. Pure Appl. Math. 33 (1980), 199-211.

[G] M. Gage, Upper bounds for the first Dirichlet eigenvalue of the Laplace-Beltrami operators, Indiana Univ. Math. J. 29 (1980), 897-912.

[O] R. Osserman, Global properties of minimal surfaces in $\mathbb{E}^{3}$ and $\mathbb{E}^{n}$, Ann. of Math. (2) 80 (1964), 340-364.

[S] M. Spivak, A comprehensive introduction to differential geometry, Vol. IV, Publish or Perish, Boston, MA, 1975.

[Sc] R. Schoen, Estimates for stable minimal surfaces in three dimensional manifolds, Seminar on Minimal Submanifolds, Princeton Univ. Press, Princeton, NJ, 1983.

Technische Universität Berlin, Str. Des 17 Juni 135, 1000 Berlin 12, Germany

Current address: Science Laboritories, University of Durham, Durham, DH1-3LE, United Kingdom

E-mail address: bennett.palmer@durham.ac.uk 